\section{Auswirkungen geriatrischer Interventionen}

\author{
Angesichts der Auswirkungen des demografischen Wandels in Deutschland \\ nimmt die Bedeutung von Themen des Alters und Alterns zu. Fragen nach best- \\ möglicher geriatrischer Versorgung und möglichen Interventionen stellen sich \\ mit großer Relevanz: Wie können Selbstständigkeit und Lebensqualität in \\ höchstem Maß erhalten und Risiken für Pflegebedürftigkeit verringert werden?
}

D er Stellenwert von Forschung auf dem Gebiet der Geriatrie und Gerontologie ist im Vergleich zu anderen Disziplinen der Medizin gering. Die Studie der Universität Köln aber untersuchte Verbesserungsmöglichkeiten hinsichtlich der ADL (, activities of daily living"), der Mobilität und der Kognition sowie depressiver Zustände während einer geriatrischen stationären Behandlung. Auch die Frage nach einer Form der möglichst frühen Prognose von Verbesserungspotenzialen wurde darin gestellt. Dazu wurden retrospektiv klinische Daten von 646 überwiegend multimorbiden Patient/-innen im Alter von $>80$ Jahren analysiert.

Ziel der Arbeit war die Erfassung des direkten Zusammenhanges potentieller Fortschritte und geriatrischer Intervention. Das heißt, einer Behandlung von typischen beziehungsweise oft auftretenden Erkrankungen, wie Herzerkrankungen, in Verbindung mit Maßnahmen zur Erhaltung oder Wiedererlangung von patienteneigenen Ressourcen. Geriatrische Assessments dienten dabei der multidimensionalen Erfassung des IstZustandes der Patient/-innen. Neben Fragebögen kamen auch Instrumente wie der Barthel Index (BI), der Mobilitätstest nach Tinetti, der Mini-Mental Status-Test, der Uhren-Test sowie die geriatrische Depressionsskala (GDS) zum Einsatz.

Durch die Ermittlung eines funktionellen, mobilen und kognitiven Status bei Aufnahme der Patienten war es möglich, entsprechende Vergleichswerte bei der Entlassung zu erheben und Fortschritte sichtbar zu machen. Nachweislich existierten Fortschritte nach erfolgter geriatrischer stationärer Behandlung im Sinne der funktionellen Erholung mit einer Verbesserung beziehungsweise Wiedererlangung von Mobilität und Selbstständigkeit. Verbesserungen gab es sowohl im Bereich der ADL als auch im Bereich der Kognition.

Laut Studie profitieren geriatrische Patient/-innen langfristig von speziell auf sie abgestimmten Behandlungsformen, die den Fokus auf Empowerment setzen. Für ein weitreichendes Verständnis von Faktoren der geriatrischen Rehabilitation sind allerdings Anschlussstudien nötig.

(dd)

S. Bordne, R.-J. Schultz, S. Zank. Effects of inpatient geriatric interventions in a German geriatric hospital Impact on ADL, mobility and cognitive status. Z Gerontol Geriat 2015 48:370-375

\section{Kommentar}

Die Struktur der Bevölkerung in den Industriestaaten ist einer stürmischen Veränderung unterworfen. Der Anteil älterer Menschen steigt erheblich, und unsere Lebenserwartung steigt jedes Jahr um drei Monate. Dies kann als großer Erfolg der modernen Medizin angesehen werden, bringt aber auch Herausforderungen mit sich. Besonders die Gesundheitsversorgung sieht sich mit neuen Problemen konfrontierten. Die immer älteren Patienten leiden unter vielen Krankheiten gleichzeitig (Multimorbidität) und die Mehrzahl dieser Erkrankungen sind chronisch. Das bedeutet: langfristige Konzepte sind unverzichtbar. Gefragt ist also zunehmend eine Medizin, die ein ganzheitliches Gesundheitsmanagement bieten kann, das sich im Idealfall auch in der Zeit nach einem akuten Krankenhausaufenthalt bewährt. Die umfassendsten Konzepte für die betroffenen Patienten bietet die Geriatrie an. Allerdings gibt es wenig wissenschaftliche Evidenz für die Wirksamkeit dieser medizinischen Maßnahmen. Eine wichtige Studie zu diesem Thema wurde jetzt in der Zeitschrift für Gerontologie und Geriatrie publiziert. Dabei wurden die Effektivität der Behandlung in einer geriatrischen Klinik untersucht und auch Langzeiteffekte (bis zu fünf Monaten) beschrieben. In einer größeren Stichpro- be $(\mathrm{N}=646)$ untersuchten die Autoren Parameter, die für die Lebensqualität der betroffenen Patienten eine wichtige Rolle spielen: Mobilität, Selbsthilfestatus, Gedächtnis und Orientierung. Es konnte gezeigt werden, dass sich nach einer komplexen geriatrischen Intervention nicht nur die erwähnten Variablen positiv verändern, sondern auch, dass dieser Effekt in den Monaten nach der Krankenhausbehandlung anhält. Das ist ein wichtiges Ergebnis, das die Diskussion zum Thema beleben wird.

Was allerdings noch fehlt, sind Studien mit einer Kontrollgruppe, die ohne das erwähnte geriatrische Gesundheitsmanagement auskommen muss. Außerdem sind die ökonomischen Effekte, die unter anderem in der Schleswig-Holstein Studie (1995) beschrieben wurden, nicht thematisiert worden. Es gibt nämlich gute Argumente für die Annahme, dass außer der Lebensqualität der Betroffenen durch die geriatrische Intervention mittelfristig auch Geld gespart wird. Erreicht wird dies durch die "Investitionen in die Selbstständigkeit der Patienten". Auf die Versorgungsforschung warten noch viele Aufgaben.

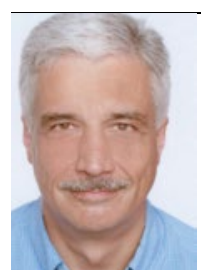
Prof. Dr. med.
Rainer Neubart Kreiskrankenhaus Wolgast gGmbH Zentrum für Altersmedizin Chausseestraße 46 17438 Wolgast geriatrie@kreiskranken- haus-wolgast.de

\section{NEUE STUDIEN}

Im August erschien die neue Ausgabe von HeilberufeSCIENCE auf SpringerLink. Neben einem Beitrag zur Übertragung heilkundlicher Tätigkeiten auf Gesundheits- und Krankenpfleger oder einer Studie zur Erwartungshaltung, Kommunikation und Kooperation von Pflegenden und Ärzten in der stationären Altenpflege, geht es in dem online-Journal auch um die Analyse der Verpflegungssituation von Kindergartenkindern. Schauen Sie doch mal rein!

http://link.springer.com/journal/16024/6/3 